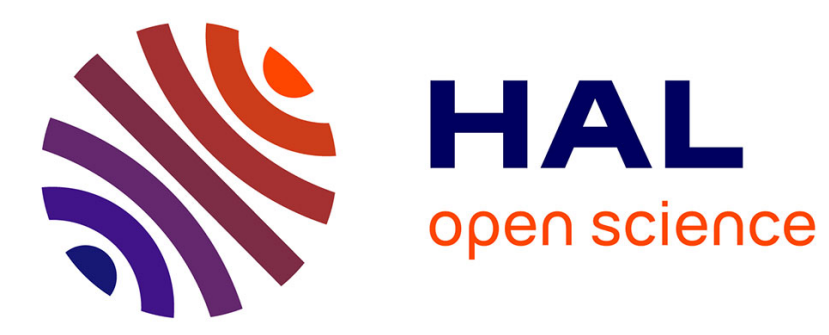

\title{
Optimal PI controller rejecting disturbance for ARZ traffic model
}

Lina Guan, Liguo Zhang, Christophe Prieur

\section{To cite this version:}

Lina Guan, Liguo Zhang, Christophe Prieur. Optimal PI controller rejecting disturbance for ARZ traffic model. CDC 2020 - 59th IEEE Conference on Decision and Control, Dec 2020, Jeju Island (virtual), South Korea. 10.1109/CDC42340.2020.9304249 . hal-03192478

\section{HAL Id: hal-03192478 https://hal.science/hal-03192478}

Submitted on 8 Apr 2021

HAL is a multi-disciplinary open access archive for the deposit and dissemination of scientific research documents, whether they are published or not. The documents may come from teaching and research institutions in France or abroad, or from public or private research centers.
L'archive ouverte pluridisciplinaire HAL, est destinée au dépôt et à la diffusion de documents scientifiques de niveau recherche, publiés ou non, émanant des établissements d'enseignement et de recherche français ou étrangers, des laboratoires publics ou privés. 


\title{
Optimal PI controller rejecting disturbance for ARZ traffic model
}

\author{
Lina Guan, Liguo Zhang and Christophe Prieur
}

\begin{abstract}
Traffic control of congestion regimes is considered in this paper. A perturbed distributed parameter model is used, and a boundary control is designed to reject the perturbations. More precisely an optimal proportional-integral (PI) feedback control law is computed to maximally reject the disturbances, and to stabilize the traffic in congested regime. The disturbance applies at the boundary of the linearized Aw-RascleZhang (ARZ) model. Therefore the disturbance operator is unbounded, rendering the control problem very challenging. In order to analyze and design the optimal PI controller for this infinite-dimensional system, the $\mathcal{L}_{2}$ gain is computed to estimate the disturbance rejection. Numerically tractable conditions are computed and written with linear matrix inequalities (LMIs). As a result, the estimation of an upper bound of the $\mathcal{L}_{2}$ gain, from the disturbance to the controlled output, can be formulated as an optimization problem with LMI constraints. The validity of this method is checked on simulations of the nonlinear ARZ model in closed-loop with this optimal PI controller.
\end{abstract}

\section{INTRODUCTION}

Traffic congestion usually happens, for instance, when the number of vehicles travelling on a road exceeds the capacity of road transportation during rush hour. Congestion regime happens also when vehicles go through a bottleneck resulting from changed traffic conditions downstream such as a slowly moving oversize vehicle, a speed limit sign, or a narrower road section. Traffic congestion can cause a series of issues including additional travel time, increased fuel consumption and $\mathrm{CO}_{2}$ emission, higher traffic accident rate and so on.

In order to keep traffic flow stable, the PI feedback control strategy can be used due to its actual superiority of attenuating disturbances in engineering. In control theory, PI feedback control method is a fruitful paradigm for the industrial and real-life applications (see e.g., [1]), and for infinite-dimensional systems. Indeed, dissipative PI boundary conditions have been given for one-dimensional linear hyperbolic systems of balance laws in [3]. Lyapunov stability

Lina Guan is with Faculty of Information Technology, Beijing University of Technology, 100124, Beijing, China, Key Laboratory of Computational Intelligence and Intelligent Systems, 100124, Beijing, China, and Univ. Grenoble Alpes, CNRS, Grenoble INP, GIPSA-lab, F-38000 Grenoble, France. Lina. Guan@gipsa-lab. fr

Liguo Zhang is with Faculty of Information Technology, Beijing University of Technology, 100124, Beijing, China and Key Laboratory of Computational Intelligence and Intelligent Systems, 100124, Beijing, China. zhangliguo@bjut.edu.cn

Christophe Prieur is with Univ. Grenoble Alpes, CNRS, Grenoble INP, GIPSA-lab, F-38000 Grenoble, France. christophe.prieuregipsa-lab. fr

This work is supported by the National Natural Science Foundation of China (NSFC, Grant No. 61873007), the Beijing Natural Science Foundation (Grant No. 1182001), and a research grant from project PHC CAI YUANPEI under grant number 44029QD, and by MIAI @ Grenoble Alpes, (ANR-19P3IA-0003). of hyperbolic systems of conservation laws is achieved for boundary control law with integral action in [7]. For the stability of a cascaded network consisting of several $2 \times 2$ linear hyperbolic systems, PI output feedback controllers are designed to reject disturbance and regulate output to the desired points in [9]. A PI controller only depending on one side measurable angular velocity is used to analyze the stability of inhomogeneous drilling model in [8]. In [11], a PI boundary feedback controller is designed to stabilize the oscillations of the traffic parameters on a freeway segment by using on-ramp metering and variable speed control.

The contribution of this paper is the design of an optimal PI boundary feedback controller maximizing the disturbance rejection, and consequently strengthens the robustness of the traffic dynamics. Computing the value of $\mathcal{L}_{2}$ gain from disturbance to output is a classical control method to measure the disturbance rejection capacity (see the recent survey [6]). In the context of congestion regimes with traffic control, it is a difficult and hard problem because it is necessary to consider infinite-dimensional models as the Aw-RascleZhang (ARZ) model [2], [10]. Disturbances apply at the boundary so that the input operator is unbounded, and finite-dimensional approximations may not be sufficient to design optimal controller. In this paper we succeed to design an optimal PI controller computed on a linearized model. Our method yields numerically tractable conditions since convex conditions are derived for the optimal PI controller design. Moreover we check on numerical simulations that the nonlinear ARZ model in closed-loop with this controller is stable.

The paper is organized as follows. In Section II, the linearized ARZ traffic flow model around an uniform steady state is presented and a PI boundary feedback controller with disturbance term is derived. The LMI conditions for finite-gain $\mathcal{L}_{2}$ stability and an upper bound of the $\mathcal{L}_{2}$ gain are given in Section III. In Section IV, the estimation of an upper bound of the $\mathcal{L}_{2}$ gain is addressed in terms of optimization problem with LMI constraints, and the results of the numerical simulation verify the validity of this method. Conclusion is given in Section V.

\section{LINEARIZED ARZ TRAFFIC FLOW MODEL}

From a macroscopic perspective, the Aw-Rascle-Zhang model describes the traffic flow dynamics as

$$
\begin{gathered}
\partial_{t} \rho+\partial_{x}(v \rho)=0, \\
\partial_{t} v+\left(v-\rho p^{\prime}(\rho)\right) \partial_{x} v=\frac{G(\rho)-v}{\tau},
\end{gathered}
$$

with an independent space variable $x$ on a finite interval $(0, L)$ and an independent time variable $t$ in $[0, \infty)$, where 


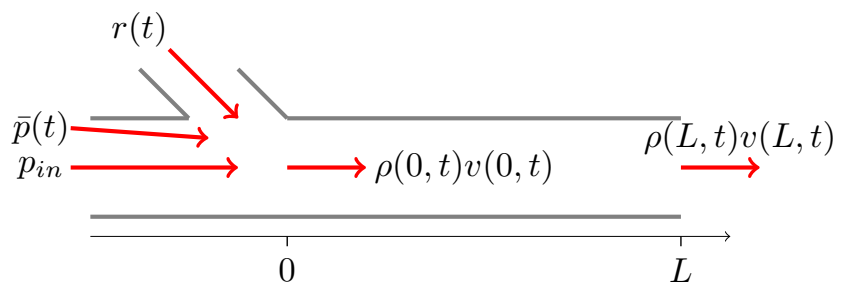

Fig. 1. The traffic flow in a freeway segment.

$\rho(x, t)$ is the vehicle density, $v(x, t)$ is the average speed, $\tau$ is the relaxation time subject to driving behavior, and $L$ is the length of the considered road segment.

The speed-density relation function $G(\rho)$ is given by the Greenshields model in [4],

$$
G(\rho)=v_{f}\left(1-\frac{\rho}{\rho_{m}}\right)
$$

where $v_{f}$ is the free flow speed, and $\rho_{m}$ is the maximum density.

The traffic pressure $p(\rho)$ is defined as follows,

$$
p(\rho)=v_{f}-G(\rho)=\frac{v_{f}}{\rho_{m}} \rho .
$$

Let $\omega=v+\frac{v_{f}}{\rho_{m}} \rho$, then (1)-(2) can be rewritten as

$$
\partial_{t}\left[\begin{array}{l}
\omega \\
v
\end{array}\right]+\left[\begin{array}{cc}
v & 0 \\
0 & 2 v-\omega
\end{array}\right] \partial_{x}\left[\begin{array}{l}
\omega \\
v
\end{array}\right]=\left[\begin{array}{c}
\frac{v_{f}-\omega}{\tau} \\
\frac{v_{f}-\omega}{\tau}
\end{array}\right] .
$$

Assume that this system is strictly hyperbolic and lies in the traffic congestion regime, i.e., the sign of the characteristic value $2 v-\omega$ in equation (5) is negative.

Let $\left(\omega^{*}, v^{*}\right)^{\top}$ be an uniform steady state of the system (5), define $\omega^{*}=v_{f}$ and select the constant $v^{*}$ such that $2 v^{*}-\omega^{*}<0$, then the following linearized ARZ model can be derived,

$$
\partial_{t}\left[\begin{array}{c}
\widetilde{\omega} \\
\widetilde{v}
\end{array}\right]+\left[\begin{array}{cc}
\lambda_{1} & 0 \\
0 & -\lambda_{2}
\end{array}\right] \partial_{x}\left[\begin{array}{l}
\widetilde{\omega} \\
\widetilde{v}
\end{array}\right]=\left[\begin{array}{cc}
-\frac{1}{\tau} & 0 \\
-\frac{1}{\tau} & 0
\end{array}\right]\left[\begin{array}{c}
\widetilde{\omega} \\
\widetilde{v}
\end{array}\right],
$$

where $\widetilde{\omega}=\omega-\omega^{*}, \widetilde{v}=v-v^{*}$, with the constant characteristic velocities

$$
\begin{gathered}
\lambda_{1}=v^{*}, \\
-\lambda_{2}=2 v^{*}-\omega^{*} .
\end{gathered}
$$

According to the flow conservation at the inlet (see Fig. 1), we have

$$
p_{i n}+\bar{p}(t)+r(t)=\rho(0, t) v(0, t)
$$

where $p_{i n}$ is the constant traffic demand, $\bar{p}(t)$ is the fluctuation of the flow rate resulting from physical perturbation, and $r(t)$ is on-ramp metering. Define nominal flux rate $r^{*}$ satisfying the relation $p_{i n}+r^{*}=\rho^{*} v^{*}$, here density $\rho^{*}$ is given by $\rho^{*}=\frac{\rho_{m}}{v_{f}}\left(\omega^{*}-v^{*}\right)$.
The following PI boundary feedback control law is introduced, for all $t \neq 0$,

$$
\begin{aligned}
r(t)=r^{*} & +k_{P 1}\left(\rho(L, t)-\rho^{*}\right) \\
+ & k_{I 1} \int_{0}^{t}\left(\rho(L, \sigma)-\rho^{*}\right) d \sigma \\
v(L, t)= & v^{*}+k_{P 2}\left(v(0, t)-v^{*}\right) \\
& +k_{I 2} \int_{0}^{t}\left(v(0, \sigma)-v^{*}\right) d \sigma
\end{aligned}
$$

where the inflow speed $v(0, t)$ and the outflow density $\rho(L, t)$ are measurement outputs, the inlet on-ramp metering $r(t)$ and the outlet variable speed $v(L, t)$ are boundary control inputs, and $k_{P 1}, k_{I 1}, k_{P 2}, k_{I 2}$ are proportional and integral tuning gains.

Let $\widetilde{\rho}=\rho-\rho^{*}$, then (10)-(11) can be rewritten as

$$
\begin{gathered}
r(t)=r^{*}+k_{P 1} \widetilde{\rho}(L, t)+k_{I 1} \int_{0}^{t} \widetilde{\rho}(L, \sigma) d \sigma, \\
\widetilde{v}(L, t)=k_{P 2} \widetilde{v}(0, t)+k_{I 2} \int_{0}^{t} \widetilde{v}(0, \sigma) d \sigma .
\end{gathered}
$$

Combining feedback control laws (12)-(13) with (9) and linearizing, the following boundary condition can be attained,

$$
\begin{aligned}
& \bar{p}(t)+k_{P 1} \widetilde{\rho}(L, t)+k_{I 1} \int_{0}^{t} \widetilde{\rho}(L, \sigma) d \sigma \\
& =v^{*} \widetilde{\rho}(0, t)+\rho^{*} \widetilde{v}(0, t) .
\end{aligned}
$$

Getting rid of the high order terms, we deduce from (12) and (14),

$$
\begin{aligned}
\widetilde{\omega}(0, t)= & \widetilde{v}(0, t)+\frac{v_{f}}{\rho_{m}} \widetilde{\rho}(0, t) \\
= & \frac{v_{f} \bar{p}(t)}{\rho_{m} v^{*}}+\left(1-\frac{v_{f} \rho^{*}}{\rho_{m} v^{*}}\right) \widetilde{v}(0, t)+\frac{v_{f} k_{P 1}}{\rho_{m} v^{*}} \widetilde{\rho}(L, t) \\
& +\frac{v_{f} k_{I 1}}{\rho_{m} v^{*}} \int_{0}^{t} \widetilde{\rho}(L, \sigma) d \sigma \\
= & \frac{v_{f} \bar{p}(t)}{\rho_{m} v^{*}}+\frac{k_{P 1}}{v^{*}} \widetilde{\omega}(L, t)+\frac{k_{I 1}}{v^{*}} \int_{0}^{t} \widetilde{\omega}(L, \sigma) d \sigma \\
& +\left(1-\frac{v_{f} \rho^{*}}{\rho_{m} v^{*}}-\frac{k_{P 1} k_{P 2}}{v^{*}} \widetilde{v}(0, t)\right. \\
& -\left(\frac{k_{P 1} k_{I 2}}{v^{*}}+\frac{k_{I 1} k_{P 2}}{v^{*}}\right) \int_{0}^{t} \widetilde{v}(0, \sigma) d \sigma .
\end{aligned}
$$

Combining (13) and (15) together for the system (6), the following PI boundary feedback controller can be derived,

$$
\begin{aligned}
{\left[\begin{array}{l}
\widetilde{\omega}(0, t) \\
\widetilde{v}(L, t)
\end{array}\right]=} & K_{P}\left[\begin{array}{c}
\widetilde{\omega}(L, t) \\
\widetilde{v}(0, t)
\end{array}\right]+K_{I} \int_{0}^{t}\left[\begin{array}{c}
\widetilde{\omega}(L, \sigma) \\
\widetilde{v}(0, \sigma)
\end{array}\right] d \sigma \\
& +\theta(t)
\end{aligned}
$$

where

$$
K_{P}=\left[\begin{array}{cc}
\frac{k_{P 1}}{v^{*}} & 1-\frac{v_{f} \rho^{*}}{\rho_{m} v^{*}}-\frac{k_{P 1} k_{P 2}}{v^{*}} \\
0 & k_{P 2}
\end{array}\right]
$$

and

$$
K_{I}=\left[\begin{array}{cc}
\frac{k_{I 1}}{v^{*}} & \frac{-k_{P 1} k_{I 2}-k_{I 1} k_{P 2}}{v^{*}} \\
0 & k_{I 2}
\end{array}\right]
$$


are respectively proportional and integral tuning matrices, and

$$
\theta(t)=\left[\begin{array}{ll}
\frac{v_{f} \bar{p}(t)}{\rho_{m} v^{*}} & 0
\end{array}\right]^{\top}
$$

is an external disturbance input vector. Assume the tuning parameters $k_{I 1}$ and $k_{I 2}$ are different to 0 , so that the matrix $K_{I}$ is invertible.

Letting $R(x, t)=(\widetilde{\omega}(x, t), \widetilde{v}(x, t))^{\top}$, for all $t$ in $[0,+\infty)$ and all $x$ in $[0, L]$, system (6) and (16) can be rewritten as

$$
\begin{gathered}
\partial_{t} R(x, t)+\Lambda \partial_{x} R(x, t)=M R(x, t), \\
R_{\text {in }}(t)=K_{P} R_{\text {out }}(t)+K_{I} \int_{0}^{t} R_{\text {out }}(\sigma) d \sigma+\theta(t),
\end{gathered}
$$

where

$$
\begin{gathered}
\Lambda=\operatorname{diag}\left\{\lambda_{1},-\lambda_{2}\right\}, \\
M=\left[\begin{array}{ll}
-\frac{1}{\tau} & 0 \\
-\frac{1}{\tau} & 0
\end{array}\right], \\
R_{\text {in }}(t)=(\widetilde{\omega}(0, t), \widetilde{v}(L, t))^{\top}, \\
R_{\text {out }}(t)=(\widetilde{\omega}(L, t), \widetilde{v}(0, t))^{\top} .
\end{gathered}
$$

The disturbance must be constrained by an upper limit of energy in order to avoid the boundless of the state and output. Assume that the energy of time-varying disturbance vector $\theta(t)$ is limited in $\mathcal{L}_{2}$ space by a constant positive value $\delta$ such that

$$
\begin{aligned}
& \|\theta(t)\|_{\mathcal{L}_{2}}+\|\dot{\theta}(t)\|_{\mathcal{L}_{2}} \\
& =\left(\int_{0}^{t} \theta(\sigma)^{\top} \theta(\sigma) d \sigma\right)^{\frac{1}{2}}+\left(\int_{0}^{t} \dot{\theta}^{\top}(\sigma) \dot{\theta}(\sigma) d \sigma\right)^{\frac{1}{2}} \\
& \leq \sqrt{\delta^{-1}}, \quad \forall t \geq 0 .
\end{aligned}
$$

\section{Finite-GAIN $\mathcal{L}_{2}$ StABle AND $\mathcal{L}_{2}$ GAIN}

As stated in [5], if there exist nonnegative constants $k$ and $g$ such that

$$
\|y\|_{\mathcal{L}_{2}} \leq k\|u\|_{\mathcal{L}_{2}}+g
$$

for all $u$ in $\mathcal{L}_{2}$ space, where $u$ and $y$ are respectively the system disturbance input and controlled output, and $g$ is a function of the initial condition, then the system is finite-gain $\mathcal{L}_{2}$ stable and has $\mathcal{L}_{2}$ gain less than or equal to $k$.

Let

$$
X(t)=\int_{0}^{t} R_{\text {out }}(\sigma) d \sigma+K_{I}^{-1} \theta(t),
$$

where $t$ in $[0, \infty)$, then

$$
\dot{X}(t)=R_{\text {out }}(t)+K_{I}^{-1} \dot{\theta}(t),
$$

and (21) is rewritten as

$$
R_{\text {in }}(t)=K_{P} R_{\text {out }}(t)+K_{I} X(t)
$$

Consider the initial conditions

$$
R(x, 0)=\left[\begin{array}{l}
\widetilde{\omega}(x, 0) \\
\widetilde{v}(x, 0)
\end{array}\right]=R_{0}(x),
$$

and

$$
X(0)=K_{I}^{-1} \theta(0)=K_{I}^{-1} \theta_{0}
$$

where $R_{0}(x)$ in $L^{2}(0, L), x$ in $(0, L)$ and $\theta_{0}$ in $\mathbb{R}^{2}$.

It is important to note the finite-gain $\mathcal{L}_{2}$ stability of the system and to compute the $\mathcal{L}_{2}$ gain from $(\theta(t), \dot{\theta}(t))^{\top}$ to $R_{i n}(t)$ or an upper bound of it. The following theorem presents the theoretical sufficient conditions for system (20), (29)-(30) to address this problem. The sign $*$ denotes the symmetric terms of a matrix in this paper.

Theorem 3.1: Considering the system (20), (29)-(30), if there exist positive constants $\mu$ and $\eta$, a diagonal matrix $P_{1}$ in $\mathbb{R}^{2 \times 2}$, a symmetric matrix $P_{2}$ in $\mathbb{R}^{2 \times 2}$ and a matrix $P_{3}$ in $\mathbb{R}^{2 \times 2}$ such that for all $x$ in $[0, L]$,

$$
\Omega=\left[\begin{array}{cccc}
\Omega_{11} & \Omega_{12} & \Omega_{13} & \Omega_{14} \\
* & \Omega_{22} & \Omega_{23} & O_{2} \\
* & * & \Omega_{33} & \Omega_{34} \\
* & * & * & \Omega_{44}
\end{array}\right] \geq 0,
$$

where

$$
\begin{aligned}
\Omega_{11}= & -\mu \Lambda \mathcal{P}_{1}(x)-M^{\top} \mathcal{P}_{1}(x)-\mathcal{P}_{1}(x) M \\
\Omega_{12}= & \Omega_{14}=-\mathcal{P}_{3}(x) \\
\Omega_{13}= & -\frac{\mu}{2} \Lambda \mathcal{P}_{3}(x)-M^{\top} \mathcal{P}_{3}(x) \\
\Omega_{22}= & -\frac{1}{L}\left(K_{P}^{\top}|\Lambda| P_{1} E_{3} K_{P}-|\Lambda| P_{1}\right. \\
& \left.+\frac{1}{\eta} K_{P}^{\top} K_{P}\right) \\
\Omega_{23}= & -\frac{1}{L}\left(K_{P}^{\top}|\Lambda| P_{1} E_{3} K_{I}+2 K_{P}^{\top}|\Lambda| E_{1} \mathcal{P}_{3}(0)\right. \\
& \left.-2|\Lambda| E_{2} \mathcal{P}_{3}(0)+\frac{1}{\eta} K_{P}^{\top} K_{I}\right)-P_{2} \\
\Omega_{33}= & -\frac{1}{L}\left(K_{I}^{\top}|\Lambda| P_{1} E_{3} K_{I}+2 K_{I}^{\top}|\Lambda| E_{1} \mathcal{P}_{3}(0)\right. \\
& \left.+\frac{1}{\eta} K_{I}^{\top} K_{I}\right) \\
\Omega_{34}= & -P_{2} \\
\Omega_{44}= & \frac{1}{L} I_{2}
\end{aligned}
$$

with $|\Lambda|=\operatorname{diag}\left\{\lambda_{1}, \lambda_{2}\right\}, \mathcal{P}_{1}(x)=P_{1} \operatorname{diag}\left\{e^{-\mu(L-x)}, e^{\mu x}\right\}$, $\mathcal{P}_{3}(x)=P_{3} \operatorname{diag}\left\{e^{-\frac{\mu}{2}(L-x)}, e^{\frac{\mu}{2} x}\right\}, E_{1}=\operatorname{diag}\left\{1, e^{\frac{\mu}{2} L}\right\}$, $E_{2}=\operatorname{diag}\left\{e^{\frac{\mu}{2} L}, 1\right\}, E_{3}=\left(E_{1} E_{2}^{-1}\right)^{2}$, null matrix $O_{2}$ and identity matrix $I_{2}$ are in $\mathbb{R}^{2 \times 2}$, then for all initial conditions (31) and (32), and $\theta(t)$ satisfying (26), the system (20), (29)-(30) is finite-gain $\mathcal{L}_{2}$ stable and the $\mathcal{L}_{2}$ gain from $(\theta(t), \dot{\theta}(t))^{\top}$ to $R_{\text {in }}$ is less than or equal to $\sqrt{\eta m}$ for a positive constant $m$ depending only on $K_{I}$.

Proof: The following candidate Lyapunov function is defined,

$$
\begin{aligned}
& V(R(x, t), X(t)) \\
& =\int_{0}^{L}\left[\begin{array}{c}
R(x, t) \\
X(t)
\end{array}\right]^{\top}\left[\begin{array}{cc}
\mathcal{P}_{1}(x) & \mathcal{P}_{3}(x) \\
* & P_{2}
\end{array}\right]\left[\begin{array}{c}
R(x, t) \\
X(t)
\end{array}\right] d x \\
& =\int_{0}^{L}\left[R^{\top}(x, t) \mathcal{P}_{1}(x) R(x, t)\right] d x \\
& +\int_{0}^{L}\left[R^{\top}(x, t) \mathcal{P}_{3}(x) X(t)\right. \\
& \left.+X^{\top}(t) \mathcal{P}_{3}^{\top}(x) R(x, t)\right] d x+L X^{\top}(t) P_{2} X(t) .
\end{aligned}
$$


The time derivative of $V$ along the solutions to system (20), (29)-(30) is written as follows,

$$
\dot{V}=\dot{V}_{1}+\dot{V}_{2}+\dot{V}_{3}
$$

with

$$
\begin{aligned}
\dot{V}_{1}= & \int_{0}^{L}\left[\left(\partial_{t} R\right)^{\top} \mathcal{P}_{1}(x) R+R^{\top} \mathcal{P}_{1}(x) \partial_{t} R\right] d x, \\
\dot{V}_{2}= & \int_{0}^{L}\left[\left(\partial_{t} R\right)^{\top} \mathcal{P}_{3}(x) X+R^{\top} \mathcal{P}_{3}(x) \dot{X}\right. \\
& \left.+\dot{X}^{\top} \mathcal{P}_{3}^{\top}(x) R+X^{\top} \mathcal{P}_{3}^{\top}(x) \partial_{t} R\right] d x \\
\dot{V}_{3}= & L \dot{X}^{\top} P_{2} X+L X^{\top} P_{2} \dot{X}
\end{aligned}
$$

Using (20) and performing an integration by parts in (44), the following result is achieved,

$$
\begin{aligned}
\dot{V}_{1}= & -\left.R^{\top} \Lambda \mathcal{P}_{1}(x) R\right|_{0} ^{L}+\int_{0}^{L} R^{\top}\left[\Lambda \mathcal{P}_{1}^{\prime}(x)\right. \\
& \left.+M^{\top} \mathcal{P}_{1}(x)+\mathcal{P}_{1}(x) M\right] R d x \\
= & R^{\top}(0, t) \Lambda \mathcal{P}_{1}(0) R(0, t)-R^{\top}(L, t) \Lambda \mathcal{P}_{1}(L) R(L, t) \\
& +\int_{0}^{L} R^{\top}\left[\mu \Lambda \mathcal{P}_{1}(x)+M^{\top} \mathcal{P}_{1}(x)\right. \\
& \left.+\mathcal{P}_{1}(x) M\right] R d x .
\end{aligned}
$$

From (24) and (25), previous equation (47) can be rewritten as,

$$
\begin{aligned}
\dot{V}_{1}= & R_{\text {out }}^{\top}(t)\left(K_{P}^{\top}|\Lambda| P_{1} E_{3} K_{P}-|\Lambda| P_{1}\right) R_{\text {out }}(t) \\
& +R_{\text {out }}^{\top}(t) K_{P}^{\top}|\Lambda| P_{1} E_{3} K_{I} X \\
& +X^{\top} K_{I}^{\top}|\Lambda| P_{1} E_{3} K_{P} R_{\text {out }}(t) \\
& +X^{\top} K_{I}^{\top}|\Lambda| P_{1} E_{3} K_{I} X+\int_{0}^{L} R^{\top}\left[\mu \Lambda \mathcal{P}_{1}(x)\right. \\
& \left.+M^{\top} \mathcal{P}_{1}(x)+\mathcal{P}_{1}(x) M\right] R d x
\end{aligned}
$$

From (20) and (29), the following result can be derived from (45) by using an integration by parts,

$$
\begin{aligned}
\dot{V}_{2}= & {\left.\left[-R^{\top} \Lambda \mathcal{P}_{3}(x) X-X^{\top} \mathcal{P}_{3}^{\top}(x) \Lambda R\right]\right|_{0} ^{L} } \\
& +\int_{0}^{L}\left[\frac{\mu}{2} R^{\top} \Lambda \mathcal{P}_{3}(x) X+\frac{\mu}{2} X^{\top} \mathcal{P}_{3}^{\top}(x) \Lambda R\right. \\
& +R^{\top} \mathcal{P}_{3}(x) R_{\text {out }}(t)+R^{\top} \mathcal{P}_{3}(x)\left(K_{I}^{-1} \dot{\theta}(t)\right) \\
& +\left(K_{I}^{-1} \dot{\theta}(t)\right)^{\top} \mathcal{P}_{3}^{\top}(x) R+R_{\text {out }}^{\top}(t) \mathcal{P}_{3}^{\top}(x) R \\
& \left.+R^{\top} M^{\top} \mathcal{P}_{3}(x) X+X^{\top} \mathcal{P}_{3}^{\top}(x) M R^{\prime}\right] d x \\
= & R^{\top}(0, t) \Lambda \mathcal{P}_{3}(0) X-R^{\top}(L, t) \Lambda \mathcal{P}_{3}(L) X \\
& +X^{\top} \mathcal{P}_{3}^{\top}(0) \Lambda R(0, t)-X^{\top} \mathcal{P}_{3}^{\top}(L) \Lambda R(L, t) \\
& +\int_{0}^{L}\left[\frac{\mu}{2} R^{\top} \Lambda \mathcal{P}_{3}(x) X+\frac{\mu}{2} X^{\top} \mathcal{P}_{3}^{\top}(x) \Lambda R\right. \\
& +R^{\top} \mathcal{P}_{3}(x) R_{\text {out }}(t)+R^{\top} \mathcal{P}_{3}(x)\left(K_{I}^{-1} \dot{\theta}(t)\right) \\
& +R_{\text {out }}^{\top}(t) \mathcal{P}_{3}^{\top}(x) R+\left(K_{I}^{-1} \dot{\theta}(t)\right)^{\top} \mathcal{P}_{3}^{\top}(x) R \\
& \left.+R^{\top} M^{\top} \mathcal{P}_{3}(x) X+X^{\top} \mathcal{P}_{3}^{\top}(x) M R^{\prime}\right] d x .
\end{aligned}
$$

From (24) and (25), previous equation (49) can be rewritten as,

$$
\begin{aligned}
\dot{V}_{2}= & 2 R_{\text {out }}^{\top}(t) K_{P}^{\top}|\Lambda| E_{1} \mathcal{P}_{3}(0) X \\
& +2 X^{\top} K_{I}^{\top}|\Lambda| E_{1} \mathcal{P}_{3}(0) X-2 R_{\text {out }}^{\top}(t)|\Lambda| E_{2} \mathcal{P}_{3}(0) X \\
& +\int_{0}^{L}\left[\frac{\mu}{2} R^{\top} \Lambda \mathcal{P}_{3}(x) X+\frac{\mu}{2} X^{\top} \mathcal{P}_{3}^{\top}(x) \Lambda R\right. \\
& +R^{\top} \mathcal{P}_{3}(x) R_{\text {out }}(t)+R^{\top} \mathcal{P}_{3}(x)\left(K_{I}^{-1} \dot{\theta}(t)\right) \\
& +R_{\text {out }}^{\top}(t) \mathcal{P}_{3}^{\top}(x) R+\left(K_{I}^{-1} \dot{\theta}(t)\right)^{\top} \mathcal{P}_{3}^{\top}(x) R \\
& \left.+R^{\top} M^{\top} \mathcal{P}_{3}(x) X+X^{\top} \mathcal{P}_{3}^{\top}(x) M R\right] d x
\end{aligned}
$$

From (29), we can convert (46) into

$$
\begin{aligned}
\dot{V}_{3}= & L R_{\text {out }}^{\top}(t) P_{2} X+L\left(K_{I}^{-1} \dot{\theta}(t)\right)^{\top} P_{2} X \\
& +L X^{\top} P_{2} R_{\text {out }}(t)+L X^{\top} P_{2}\left(K_{I}^{-1} \dot{\theta}(t)\right) .
\end{aligned}
$$

Then from (48), (50) and (51), the following result can be derived,

$$
\begin{aligned}
& \dot{V}+\frac{1}{\eta} R_{\text {in }}^{\top}(t) R_{\text {in }}(t)-\theta^{\top}(t) \theta(t) \\
& -\dot{\theta}^{\top}(t)\left(K_{I}^{-1}\right)^{\top} K_{I}^{-1} \dot{\theta}(t) \\
& \leq-\int_{0}^{L}\left[\begin{array}{c}
R \\
R_{\text {out }}(t) \\
X \\
K_{I}^{-1} \dot{\theta}(t)
\end{array}\right] \quad \Omega\left[\begin{array}{c}
R \\
R_{\text {out }}(t) \\
X \\
K_{I}^{-1} \dot{\theta}(t)
\end{array}\right] d x,
\end{aligned}
$$

where $\Omega$ satisfies (33).

Thus, for any $\theta(t)$ satisfying (26),

$$
\begin{aligned}
\dot{V} \leq & -\frac{1}{\eta} R_{i n}^{\top}(t) R_{i n}(t)+\theta^{\top}(t) \theta(t) \\
& +\dot{\theta}^{\top}(t)\left(K_{I}^{-1}\right)^{\top} K_{I}^{-1} \dot{\theta}(t) .
\end{aligned}
$$

By integrating the both sides of the previous inequality (53) over the interval $[0, t]$, one can derive that

$$
\begin{aligned}
& \int_{0}^{t} \dot{V}(R(x, \sigma), X(\sigma)) d \sigma \\
& \leq-\frac{1}{\eta} \int_{0}^{t} R_{i n}^{\top}(\sigma) R_{i n}(\sigma) d \sigma+m \int_{0}^{t} \theta^{\top}(\sigma) \theta(\sigma) d \sigma \\
& \quad+m \int_{0}^{t} \dot{\theta}^{\top}(\sigma) \dot{\theta}(\sigma) d \sigma
\end{aligned}
$$

where $m=\max \left\{1, \lambda_{\max }\left(\left(K_{I}^{-1}\right)^{\top} K_{I}^{-1}\right)\right\}$. Then from $V(R(x, t), X(t)) \geq 0$,

$$
\begin{aligned}
& \int_{0}^{t} R_{i n}^{\top}(\sigma) R_{i n}(\sigma) d \sigma \\
& \leq \eta V\left(R_{0}(x), X(0)\right)+\eta m \int_{0}^{t} \theta^{\top}(\sigma) \theta(\sigma) d \sigma \\
& \quad+\eta m \int_{0}^{t} \dot{\theta}^{\top}(\sigma) \dot{\theta}(\sigma) d \sigma .
\end{aligned}
$$

Using the inequality $\alpha^{2}+\beta^{2} \leq(\alpha+\beta)^{2}$ for nonnegative numbers $\alpha$ and $\beta$ and taking the square roots, one can obtain that

$$
\begin{aligned}
\left\|R_{\text {in }}(t)\right\|_{\mathcal{L}_{2}} \leq & \sqrt{\eta V\left(R_{0}(x), X(0)\right)} \\
& +\sqrt{\eta m}\left(\|\theta(t)\|_{\mathcal{L}_{2}}+\|\dot{\theta}(t)\|_{\mathcal{L}_{2}}\right)
\end{aligned}
$$


where the bias term $\sqrt{\eta V\left(R_{0}(x), X(0)\right)}$ depends on the initial conditions $R_{0}(x)$ and $X(0)$.

Note that if $R_{0}(x)=0$ and $\theta_{0}=0$,

$$
\left\|R_{\text {in }}(t)\right\|_{\mathcal{L}_{2}} \leq \sqrt{\eta m}\left(\|\theta(t)\|_{\mathcal{L}_{2}}+\|\dot{\theta}(t)\|_{\mathcal{L}_{2}}\right) .
$$

Therefore, the system (20), (29)-(30) is finite-gain $\mathcal{L}_{2}$ stable and has $\mathcal{L}_{2}$ gain which is less than or equal to $\sqrt{\eta m}$.

\section{NUMERICAL ISSUES}

\section{A. Optimization}

The smaller value of the $\mathcal{L}_{2}$ gain, the stronger the capacity of disturbance rejection is. Based on Theorem 3.1, the following optimization problem can be considered to estimate $\mathcal{L}_{2}$ gain,

$$
\min _{P_{1}, P_{2}, P_{3}, \mu} \eta
$$

subject to relations (33).

\section{B. Simulations}

In order to seek the optimal values of parameter matrices $K_{P}, K_{I}$ through the numerical simulation of optimization problem, we consider a road segment with parameters, $\rho_{m}=$ 213.3 veh. $/ \mathrm{km}, v_{f}=160 \mathrm{~km} / \mathrm{h}, L=1 \mathrm{~km}$, and $\tau=60 \mathrm{~s}$. The initial conditions are

$$
\begin{aligned}
& \rho(x, 0)=\rho^{*}+0.8 \sin 4 \pi x, \\
& v(x, 0)=v^{*}+1.8 \cos 4 \pi x,
\end{aligned}
$$

where the steady state $\left(\rho^{*}, v^{*}\right)^{\top}=(120,70)^{\top}$ satisfies the ARZ equations (1) and (2). With given $\delta=0.6$ in (26), the disturbance $\bar{p}(t)$ is given as Fig. 2.

By solving optimization problem (58), we can derive the relation between $\eta_{\min }$ (the minimal value of $\eta$ ) and $\mu$ in Fig. 3. In particular, for the value of $\eta_{\min }=1.1113 \times$ $10^{10}, \mu=0.09$ should be selected. Moreover, we solve the optimization problem by computing the best tuning parameters $k_{P 1}, k_{P 2}, k_{I 1}, k_{I 2}$, and we get $k_{P 1}=-20$, $k_{P 2}=-0.1, k_{I 1}=-20, k_{I 2}=-0.5$, and $m=14.2518$. The corresponding parameter matrices $K_{P}$ and $K_{I}$ of PI boundary controller in (16) are

$$
\begin{aligned}
K_{P} & =\left[\begin{array}{cc}
-0.2857 & -0.3143 \\
0 & -0.1
\end{array}\right], \\
K_{I} & =\left[\begin{array}{cc}
-0.2857 & -0.1714 \\
0 & -0.5
\end{array}\right] .
\end{aligned}
$$

Under the optimal values of $K_{P}, K_{I}$, the state $(\widetilde{\omega}, \widetilde{v})^{\top}$ of the linearized ARZ traffic system (20) converges to the zero steady state, as seen in Fig. 4 and Fig. 5. In Fig. 6, the evolution of the two components of $R_{i n}(t)$ given by (30) tends to be stable as time goes. We can observe in Fig. 7 and Fig. 8 that the state $(\rho, v)^{\top}$ of the nonlinear ARZ system (1)-(2) exponentially converges to the steady state $\left(\rho^{*}, v^{*}\right)^{\top}$.

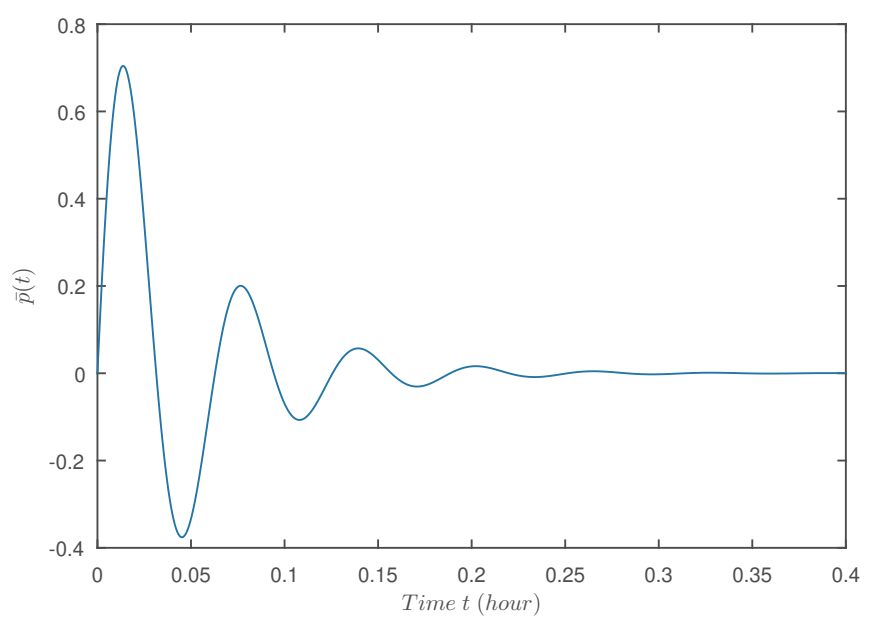

Fig. 2. Evolution of the disturbance $\bar{p}(t)$.

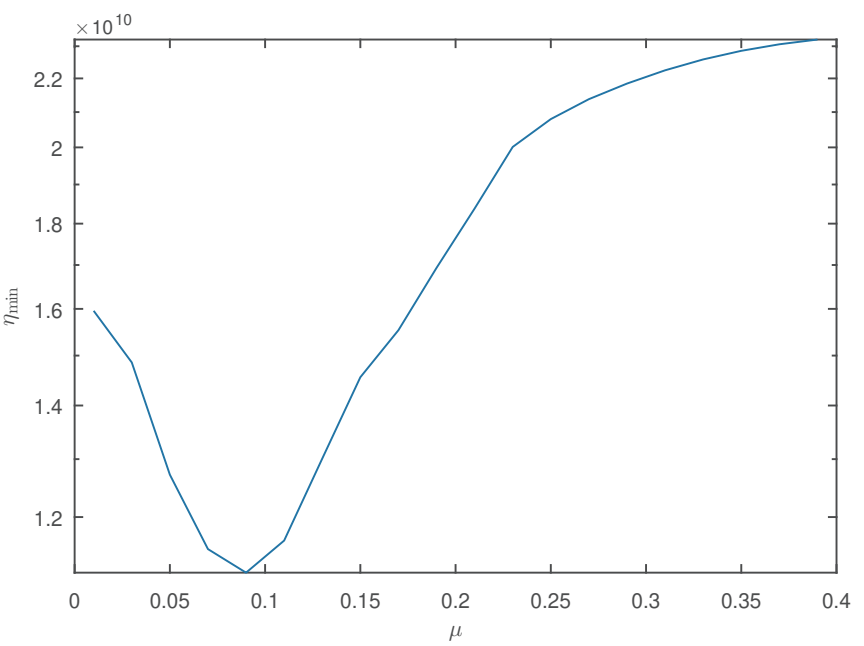

Fig. 3. Relation between $\eta_{\min }$ and $\mu$.

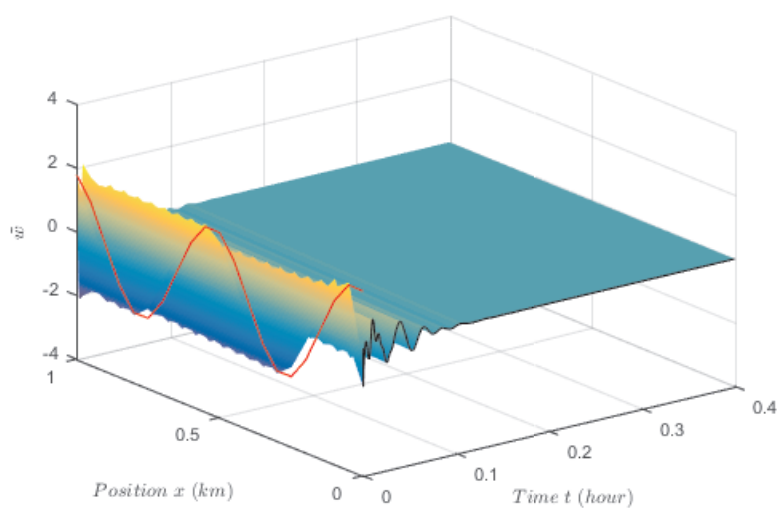

Fig. 4. State $\widetilde{\omega}$ of linearized ARZ traffic system (20), (29)-(30).

\section{CONCLUSion}

This paper addressed the problem of seeking the optimal PI boundary feedback controller to maximize the capacity of 


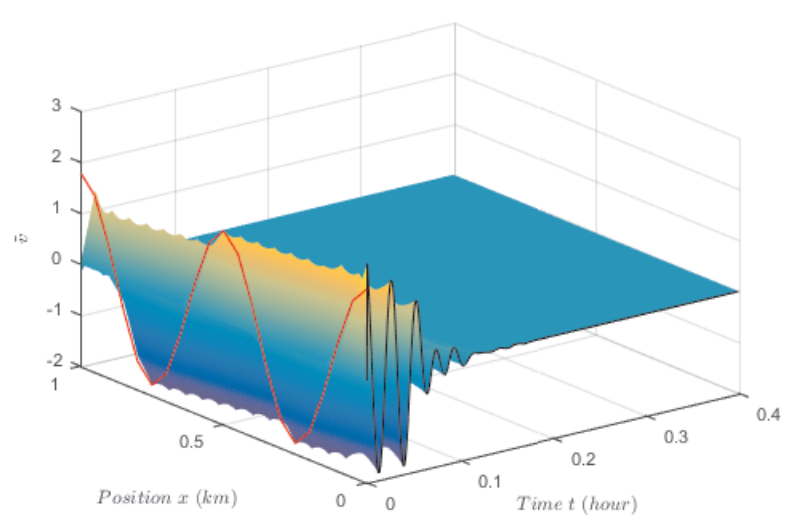

Fig. 5. State $\widetilde{v}$ of linearized ARZ traffic system (20), (29)-(30).
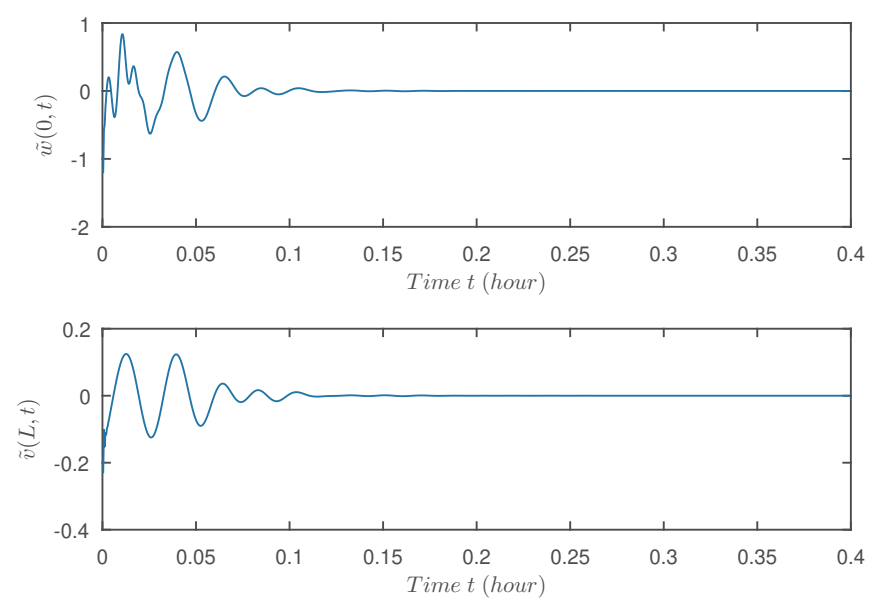

Fig. 6. Time evolution of the two components of $R_{i n}(t)$ given by (24).

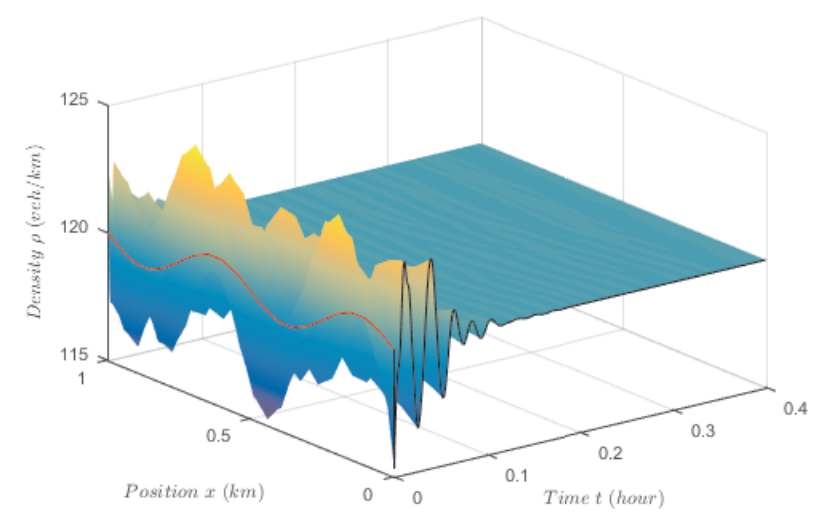

Fig. 7. State $\rho$ of nonlinear ARZ system (1)-(2).

disturbance rejection for the linear traffic flux system. The conditions for finite-gain $\mathcal{L}_{2}$ stability and the computation of the $\mathcal{L}_{2}$ gain were given for the linearized ARZ system. Based on these conditions, we formulated and solved an optimization problem to derive the optimal PI controller.

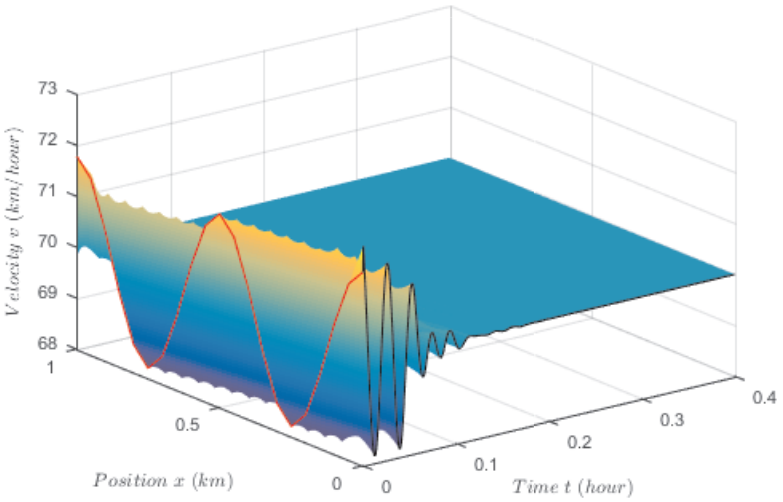

Fig. 8. State $v$ of nonlinear ARZ system (1)-(2).

Theoretical result states the disturbance rejection for the linearized system. Moreover numerical simulation emphasize the interest of this optimal controller for the nonlinear model.

Many optimization problems of traffic flux systems remain open. The class of nonlinear traffic flux systems with $\mathcal{L}_{2}$ disturbance or other disturbance measurement methods could be studied to maximize the disturbance rejection capacity. The extension of optimization problem to the network of roads could be also of interest.

\section{REFERENCES}

[1] Kiam Heong Ang, Gregory Chong, and Yun Li. PID control system analysis, design, and technology. IEEE transactions on control systems technology, 13(4):559-576, 2005.

[2] A. Aw and M. Rascle. Resurrection of "second order" models of traffic flow. SIAM J. Appl. Math., 60(3):916-938, 2000.

[3] Ababacar Diagne, Georges Bastin, and Jean-Michel Coron. Lyapunov exponential stability of 1-D linear hyperbolic systems of balance laws. Automatica, 48:109-114, 2012.

[4] BD Greenshields, JR Bibbins, WS Channing, and HH Miller. A study of traffic capacity. Highway research board proceedings, 1935, 1935.

[5] Hassan K Khalil and Jessy W Grizzle. Nonlinear systems, volume 3. Prentice hall Upper Saddle River, NJ, 2002.

[6] Andrii Mironchenko and Christophe Prieur. Input-to-state stability of infinite-dimensional systems: recent results and open questions. SIAM Review, 62(3):529-614, 2020.

[7] V. Dos Santos, G. Bastin, J.-M. Coron, and B. d'Andréa Novel. Boundary control with integral action for hyperbolic systems of conservation laws: Stability and experiments. Automatica, 44:13101318,2008

[8] Alexandre Terrand-Jeanne, Vincent Andrieu, Melaz Tayakout-Fayolle, and Valérie dos Santos Martins. Regulation of inhomogeneous drilling model with a P-I controller. IEEE Transactions on Automatic Control, 65(1):58-71, 2020.

[9] Ngoc-Tu Trinh, Vincent Andrieu, and Cheng-Zhong Xu. Output regulation for a cascaded network of $2 \times 2$ hyperbolic systems with PI controller. Automatica, 91:270-278, 2018.

[10] H. M. Zhang. A non-equilibrium traffic model devoid of gas-like behavior. Transportation Research Part B, 36(3):275-290, 2002.

[11] Liguo Zhang, Christophe Prieur, and Junfei Qiao. PI boundary control of linear hyperbolic balance laws with stabilization of ARZ traffic flow models. Systems \& Control Letters, 123:85-91, 2019. 